\section{Cahiers de Narratologie}

Analyse et théorie narratives

8 | 1997

Création de l'espace et narration littéraire

\title{
Indices et symboles
}

L'espace d'une initiation

\section{Anna Jaubert}

\section{CpenEdition}

Journals

Édition électronique

URL : http://journals.openedition.org/narratologie/10751

DOI : 10.4000/narratologie.10751

ISSN : 1765-307X

Éditeur

LIRCES

\section{Édition imprimée}

Date de publication : 1 décembre 1997

Pagination : 157-175

ISBN : 291089746X

ISSN : 0993-8516

\section{Référence électronique}

Anna Jaubert, «Indices et symboles », Cahiers de Narratologie [En ligne], 8 | 1997, mis en ligne le 01 décembre 2020, consulté le 25 février 2021. URL : http://journals.openedition.org/narratologie/10751 ; DOI : https://doi.org/10.4000/narratologie.10751

Ce document a été généré automatiquement le 25 février 2021.

Article L.111-1 du Code de la propriété intellectuelle. 


\title{
Indices et symboles
}

\author{
L'espace d'une initiation
}

\section{Anna Jaubert}

1 Gestion de l'espace, effet de réel, et narrativité entretiennent des rapports sensibles. Évidents ou ténus, ils conditionnent notre réception; je me propose d'éclairer ce processus à la lecture d'une de ces œuvres bien connues du XVIII ${ }^{\mathrm{e}}$ siècle, une œuvre de premier rang au "second rayon" que réservait Emile Henriot $^{1}$, mais qui certes ne déshonorerait pas le premier! Il s'agit des Égarements du cour et de l'esprit de Claude Crébillon. Ces «Égarements» se signalent d'abord par la lettre, un titre qui en son temps a fait mouche ${ }^{2}$, et ce titre est corroboré par un contenu manifeste, où l'espace, littéral et métaphorique, répercute à plusieurs niveaux les enjeux d'une écriture.

2 La Lettre n'est jamais indifférente. Le terme d'égarement, issu du verbe s'égarer, présuppose en soi une voie à suivre, un chemin dont on se serait écarté. Le substantif marque cependant sa prédilection pour le sens figuré, ce que confirment les deux compléments de détermination : « les égarements du cœur et de l'esprit ». Il s'agit là d'un parcours initiatique qui commence par les premiers pas mal dirigés d'un jeune homme dans le monde, ses errances et ses erreurs. Avec ces Mémoires du Chevalier de Meilcour, c'est le sous-titre de notre œuvre, le roman suit une des voies qui, depuis son accession à la réflexivité, fait de lui un antiroman ${ }^{3}$ : nous avons là une variante du roman d'éducation (ou du roman d'apprentissage), mais, et c'est l'écart qui attire l'attention, il se développe dans une remarquable économie des lieux.

3 On s'en convaincra en suivant la trace de Meilcour pendant ces quinze journées narrées, l'histoire de la tranche de vie que déroule le récit ${ }^{4}$. Cette promenade, répétitive et sans prétention au pittoresque, délimite un espace chargé de valeurs subjectives, un espace très « habité ». 


\section{Les allées et venues de l'initiant}

4 Un narrateur, je rappelle qu'il s'agit de pseudo-mémoires, raconte son « entrée dans le monde »: "J'entrai dans le monde à dix-sept ans », sont les paroles inaugurales d'un incipit bien connu que Jean Sgard a commenté ${ }^{5}$ :

(1) «J'entrai dans le monde à dix-sept ans, et avec tous les avantages qui peuvent y faire remarquer. Mon père m'avait laissé un grand nom, dont il avait lui-même augmenté l'éclat ; et j'attendais de ma mère des biens considérables... » (p. 69) ${ }^{6}$.

Cette mère au surplus est parfaite à tous égards... Mais voilà ce jeune homme bien né, de grande fortune, en proie à « l'ennui intérieur ». Pour combler le vide de sa vie, il ne songe plus qu'à se faire une passion « telle qu'elle pût être » (p. 70).

6 Une amie de sa mère, la Marquise de Lursay, s'intéresse à lui et se voudrait l'heureuse initiatrice...

7 Sur le thème de l'entrée dans le monde se greffe ainsi le motif de la femme qui «met dans le monde ». La formule désigne l'aboutissement de l'initiation, un aboutissement sur lequel précisément l'histoire s'interrompra. Dans notre texte cette formule surgit à trois reprises : deux fois dans la bouche du petit-maître Versac, promu maître à penser, à la fin reprise par Meilcour lui-même qui s'apprivoise à cette nécessité :

(2) « Pourquoi avons-nous besoin qu'une femme nous mette dans le monde ? ( (p. 222).

8 La réponse est éludée, mais on notera que Ninon de Lenclos, bien placée pour commenter l'emploi, avait rapproché l'expression de " mettre au monde ${ }^{7}$; une femme mûre, relais de la mère, confèrerait une deuxième naissance. La métaphore et la psychanalyse ont trop beau jeu: on le leur laissera pour considérer le monde en question.

Il n'offre pas grande surprise. Le lecteur de Crébillon est en terrain connu, ce monde est celui de la «bonne compagnie», voire pour parler comme l'un des personnages, de «l'extrêmement bonne compagnie» (p. 220). Nous sommes dans un univers d'aristocrates, oisifs en ce temps de paix, nous précisent également les premières pages (c'est une de ces indications qui renvoient à une référence extra-fictionnelle), d'où le «loisir dangereux» allégué par le narrateur, et l'urgence pour le jeune homme de combler cette vacuité, de prendre une place, fût-elle illusoire, dans la société. Or ce monde, signe de prochaine asphyxie peut-être, se meut dans un périmètre des plus étroits : tout se passe à Paris, de salon en salon, de l'Opéra aux Tuileries, à l'Étoile enfin pour une vue plongeante sur la fourmilière mondaine, cette foire aux vanités. Quant à la Cour, la province ou la campagne, elles n'existent que dans les coulisses de notre histoire, elles sont évoquées certes, mais jamais nous n'avons à y suivre qui que ce soit, ce statut donnera à réfléchir.

Une remarque cependant s'impose d'entrée de jeu: l'espace manifeste, très resserré, est le fruit d'une préméditation, un parti-pris du romancier, qui s'en fait un mérite :

(3) «Le Lecteur n'y trouverait plus, à la vérité, ces événements extraordinaires et tragiques, qui enlèvent l'imagination, et déchirent le cœur; plus de héros qui ne passât les mers que pour y être, à point nommé, pris par les Turcs, plus d'aventures dans le sérail, de Sultane soustraite à la vigilance des Eunuques, par quelque tour d'adresse surprenant; plus de morts imprévues, et infiniment moins de souterrains : le fait, préparé avec art, serait rendu avec naturel. On ne pécherait plus contre les convenances et la raison. » (Préface, p. 65). 
11 Il s'agit pour Crébillon de rendre au Roman, genre décrié à l'époque, une dignité véritable à sa portée. L'on mesure du même coup l'incidence critique d'un choix délibérément "anti-romanesque». N'oublions que les préfaces disent la demande de reconnaissance d'une œuvre :

(4) «le Roman si méprisé des personnes sensées, et souvent avec justice, serait peut-être celui de tous les genres qu'on pourrait rendre le plus utile, s'il était bien manié, si, au lieu de le remplir de situations ténébreuses et forcées, de héros dont les caractères et les aventures sont toujours hors du vraisemblable, on le rendait, comme la Comédie, le tableau de la vie humaine, et qu'on y censurât les vices et les ridicules » (ibid).

12 On ne peut afficher plus clairement l'obédience aux règles classiques, fondant dans le même moule éthique et esthétique. Au nom de l'utilité (le castigat ridendo mores), la vraisemblance, au nom de la vraisemblance l'unité de lieu, en l'occurrence ce Paris mondain dont on vient de citer les endroits stratégiques, et dont la carte se dessine au fil des allées et venues du personnage central.

13 L'ennui, le besoin de se faire une passion, l'attirance pour Mme de Lursay, se manifestent concrètement par la fréquentation assidue de son salon. Un tropisme qui ne se démentira pas même si, pour les besoins de l'aventure ${ }^{8}$, il se trouve assez vite concurrencé. Quand l'histoire commence, nous sommes, point de départ symbolique, dans le salon de Mme de Meilcour, la mère du jeune homme. Après une période de deux mois ou plus, résumée en substance, l'action de premier plan est déclenchée :

(5) «Un jour qu'il y avait beaucoup de monde chez Madame de Meilcour et que [Madame de Lursay] et moi avions refusé de jouer, nous nous trouvâmes assis l'un près de l'autre : cette espèce de tête-à-tête me fit frissonner... » (p. 77).

En effet, bien mis à profit par Mme de Lursay, ce tête-à-tête conduira Meilcour à se déclarer. Le récit des Égarements va suivre désormais, au jour le jour, les déplacements de l'initiant. Celui-ci, qui perçoit à contresens le comportement de la dame, s'enferre dans une conduite d'échec :

(6) «j'allai cependant chez elle le lendemain, mais tard, et à l'heure où je savais qu'elle n'y serait pas, ou que j'y trouverais beaucoup de monde » (p. 86).

Le troisième jour il y retourne, bardé de bonnes résolutions, mais il ne la trouve pas :

(7) «J'allai chez Madame de Lursay le plus tôt qu'il me fut possible l'après-dîner, et déterminé à lui jurer que je l'adorais, et à me soumettre à ce qu'il lui plairait d'ordonner de mon sort. Malheureusement pour elle, je ne la trouvai pas: mon chagrin fut extrême et, ne sachant que devenir, j'allai, en attendant l'heure de l'Opéra faire quelques visites où je portai tout l'ennui qui m'accablait. » (p. 89).

Cette phrase est de celles qui, en balisant l'espace, dévoilent les enjeux du roman, la stratégie d'une écriture : l'ennui et l'inconsistance du personnage inadapté qui reprend à son compte mécaniquement la phraséologie courtoise d'une Chevalerie dépassée, qui « ne sait que devenir », c'est-à-dire où aller, et qui sacrifie tout aussi mécaniquement au rituel mondain de son milieu : les visites, l'Opéra (ailleurs la promenade attelée sur le Cours, et les promenades pédestres aux Tuileries), le tout réglé comme du papier à musique, avec des horaires précis. Mais on remarque aussi la malice de la prolepse narrative. Le "malheureusement pour elle», insolite dans son environnement immédiat, est une modalisation du narrateur qui anticipe sur ce qui se va se passer justement à l'Opéra, théâtre de son " coup de foudre " pour une "belle inconnue ». Mme de Lursay, dont il sait maintenant que huit jours de délai pour «l'aveu de sa 
faiblesse suffisaient à sa vertu», s'est vue ainsi cruellement devancée par les événements dès le troisième jour !

Pendant deux jours Meilcour va effectivement l'oublier :

(8) « Je ne la cherchai ni ne la vis le lendemain [...]. Toute ma journée se passa à

chercher mon inconnue ; spectacles, promenades, je visitai tout.» (p. 94).

Mais soulignons au passage l'exiguité du circuit: "spectacles, promenades, je visitai tout »; ce « tout » vite dit s'entend comme vite vu !

Le $6^{\mathrm{e}}$ jour, Mme de Lursay, inquiète d'être négligée, se rend à nouveau chez Mme de Meilcour. Elle tire le jeune homme à l'écart pour une explication, et pensant avoir "rattrapé" un amoureux transi, lui fixe rendez-vous chez elle le lendemain. Mais tout à son inconnue, Meilcour décide ce lendemain-là d'aller voir Germeuil, un ami qu'il a vu aux côtés de la jeune fille, et dont il espère tirer des renseignements. Hélas, l'ami est à la campagne, et la campagne est un lieu virtuel dans ce roman, propre à faire naître tous les fantasmes :

(9) « depuis quelques jours, ils avaient disparu l'un et l'autre ; je ne doutai pas qu'il

ne fût parti avec elle. Mon amour et ma jalousie se réveillèrent... » (p. 104).

Son désarroi lui fait chercher la solitude, et ses pas le mènent aux Tuileries, assez peu fréquentées le matin. Là il rencontre pour la deuxième fois la mystérieuse belle, sans pourtant pouvoir l'aborder.

Chez Mme de Lursay en revanche, une superbe occasion lui est offerte... qu'il va gâcher pitoyablement (p. 125 et sq.). Prêt à mieux se conduire le jour suivant, le $9^{\mathrm{e}}$, on annonce chez lui l'arrivée du comte de Versac. Le petit-maître, expert en médisance, s'acharne à détruire l'image de Mme de Lursay, et, en cette fin de première partie les sentiments du naïf jeune homme s'effondrent là même où ils étaient nés, dans le salon de Madame sa mère.

2 La deuxième partie, qui n'occupe que deux demi-journées, va le projeter plus explicitement dans la société ; les lieux en fait sont les mêmes, mais les relations s'y font plus denses, on assiste en direct aux grandes manœuvres mondaines, et Meilcour découvre que son inconnue est Hortense de Théville, la fille d'une parente chez qui il se rend le lendemain. Malade de jalousie, toujours à cause de Germeuil, familier du salon, il quitte le lieu, et, livré à lui-même, désorienté une fois de plus, il se fait conduire chez Mme de Senanges, une vieille coquette qui vient de jeter sur lui son dévolu. Malgré son aversion pour la galante délabrée, il ne peut lui refuser de l'accompagner aux Tuileries.

3 C'est sur cette promenade que s'ouvre la troisième et dernière partie du roman. Les Tuileries cette fois sont arpentées à une heure de haute fréquentation; on y rencontre Versac, et au détour d'une allée, pour la grande confusion de Meilcour, Hortense accompagnée de sa mère et de Mme de Lursay.

Le lendemain, il reçoit de Madame de Lursay un billet l'invitant à une partie de campagne de deux jours; il décline cette offre, pour ne pas s'éloigner d'Hortense. Fatale erreur puisqu'à peine arrivé chez Mme de Théville, il apprend qu'Hortense est de cette partie de campagne, et comble d'infortune, Germeuil aussi ! Désespéré, il rentre se morfondre chez lui. Versac vient alors le voir et l'entraîne à l'Étoile pour un célèbre cours magistral ${ }^{9}$ :

(10) « ... pour nous livrer plus librement à des objets qui, par leur étendue et leur variété pourront nous mener loin, je voudrais que nous allassions chercher quelque promenade solitaire, où nous pussions n'être pas interrompus, et je crois que l'Étoile pourrait convenir à notre dessein. » (p. 208). 
Le $14^{\mathrm{e}}$ jour les dames sont revenues, mais ne trouvant pas Mlle de Théville chez elle, Meilcour la cherche chez Mme de Lursay; déception encore, mais par un manège bien conduit la tendre et habile Marquise réveille l'intérêt du jeune homme... et parvient cette fois à ses fins! Aube du $15^{\mathrm{e}}$ jour et interruption de l'histoire :

(11) «Grâces aux bienséances que Mme de Lursay observait sévèrement, elle me envoya enfin, et je la quittai, en lui promettant malgré mes remords, de la voir le

lendemain de bonne heure, très déterminé, de plus, à lui tenir parole. » (p. 248).

Égarements narrés et régularité du récit. D'égarement en égarement, nous constatons l'observance et la solidarité des règles classiques: l'unité de lieu accompagne un resserrement de l'action dans le temps. L'action elle-même est homogène, le récit d'initiation comportant les étapes canoniques de l'illusion, des luttes d'influence, et de l'entrée finalement dans le jeu mondain.

Le rapport avec la règle de la vraisemblance invoqué dans la Préface s'impose de même. On mesure facilement l'intérêt de cet espace restreint. Les rencontres qui font le roman, et, quand le hasard est généreux, elles font le romanesque, deviennent ici des plus plausibles ; ainsi Meilcour a bien rencontré une deuxième fois son inconnue, pour autant n'allons pas croire aux miracles : aucun faux pas à point nommé, aucune entorse bénie ne lui fournit une occasion de rapprochement ! Le narrateur s'en amuse, égratignant au passage une scène célèbre de La Vie de Marianne ${ }^{10}$. Minces sont les marges de manœuvres dans ce circuit fermé. Fermé par les conventions du microcosme mis en scène, et fermé aussi par la stratégie du texte. Ce que l'espace perd en extension, il le regagne en intension, l'intension avec $s$ étant la teneur en signification: l'espace fonctionne comme un signe dans une production narrative. Il est indice, c'est-à-dire ancrage pour la trame événementielle, il est symbole, au-delà de l'événementiel. Ce qui ouvre la deuxième partie de cet exposé.

\section{Les hauts lieux du libertinage}

Il n'y a pas ici de bas-lieux. Ce choix d'un espace est aussi celui d'un sous-genre ${ }^{11}$ Autre allégeance aux règles classiques, la bienséance est aussi scrupuleusement respectée que l'unité de lieu. Le pittoresque y perd sans doute, et la suggestivité érotique, mais l'enjeu est différent. Le récit d'initiation vise, à travers un dépucelage-prétexte, l'accès à la maîtrise du code mondain ${ }^{12}$, voire au dépassement de ce code. Meilcour jeune, naïf, désorienté au moindre contretemps, est vu tournant en rond comme dans un aquarium. La vision rétrospective du narrateur qui domine la situation d'un savoir et d'une maîtrise ultérieurement gagnés, accentue par là son effet de recul. La distanciation est aussi, par loi physique, un effet induit de la perception rapetissée des choses, qui permet d'embrasser une totalité. La vision de l'espace où se meut le «je» du passé, qualifie par contraste la place du «je » de l'écriture. Place, valeur, identité sont dans un rapport d'inclusion. Parallèlement l'intervalle temporel, lui aussi très limité, confère à la tranche de vie retenue la valeur d'un «prélèvement significatif $»$.

Il faut dès lors envisager la symbolique des lieux.

II. 1. Espaces couverts ou espaces découverts, il s'agit d'abord de lieux où l'on est vu.

C'est le but manifeste des promenades au Tuileries, en fin d'après-midi : 
(12) «L'heure du cours était passée quand nous entrâmes dans les Tuileries; le jardin était rempli de monde. Madame de Senanges, qui ne m'y menait que pour m'y montrer, en fut charmée et résolut de se comporter si bien, qu'on ne pût pas douter que je ne lui appartinsse. » des autres qui rend licite le tête-à-tête dans un endroit à la fois à l'écart et très proche

(18) « l'endroit du salon que nous occupions était désert » (p. 77),

« Madame de Lursay, après ces paroles, me quitta brusquement; et se rapprochant de la compagnie, m'ôta l'espérance de continuer l'entretien [...] J'étais toujours resté à la même place: elle revint m'y chercher, et m'excita à parler sur différents sujets... » (p. 83).

Mme de Lursay peut même se permettre d'attirer Meilcour dans une pièce plus tranquille :

(19) «à propos, Monsieur, me dit-elle, d'un air fort sérieux, j'ai à vous parler, suivez-moi : elle passa à ces mots dans une autre chambre » (p. 97).

II. 2. On distingue ainsi des lieux dans les lieux.

43 
conflit entre les lieux publics, garants présumés de la décence, et le désir d'intimité. Significatif est à cet égard le stratagème imaginé par Mme de Lursay pour que Meilcour ait prétexte à rester dans son salon après le départ de la compagnie :

(20) «Pourquoi, ne voulez-vous pas que nous nous entretenions ce soir ? Parce que, répondit-elle il y a trop de monde ici, et que la bienséance serait choquée, si l'on vous y voyait rester... » (p. 119).

Qu'importe, il suffira de donner les ordres appropriés pour que Meilcour, apparemment en panne d'équipage, puisse rester en feignant d'attendre ses gens. Il est intéressant de voir comme la leçon a porté : Meilcour, à la fin du récit, prête une fausse sortie à un rival imaginaire, et, lui opposant une fausse sortie effective, vient surprendre Mme de Lursay restée seule sur son canapé, avec cette fois la suite espérée de la situation. Un long « mauvais usage » du « sopha » :

(21) «il n'est pas hors de propos de faire remarquer que quand elle s'était remise sur le sopha, je m'étais rejeté à ses pieds; qu'alors elle m'avait laissé appuyer les coudes sur ses genoux; que d'une main elle badinait avec mes cheveux, et qu'elle permettait que je lui serrasse ou baisasse l'autre, car cette importante faveur était à mon choix...

[...] Tout ajoutait à l'occasion, et rien ne me la fit comprendre [...] Je l'aurais tourmentée jusqu'au jour de mes protestations d'amour, et surtout de respect, si, ennuyée enfin de la situation ridicule où je la mettais, elle ne m'eût réitéré, et très fortement, qu'il était temps que je me retirasse. » (pp. 126-127)),

se résout ainsi dans un subterfuge de comédie, dans une logique de « vaudeville » avant l'heure.

II. 3. D'autres lieux dans les lieux, figurent un espace espace-gigogne. En plein air ou non, ils surdéterminent l'idée de clôture qui visiblement parle à l'imaginaire érotique. À l'Opéra, c'est dans une loge que Meilcour entend savourer son vague-à-l'âme, et c'est dans une loge voisine que la belle inconnue fait son apparition, produisant une impression violente sur les sens du jeune homme. Aux Tuileries, c'est symboliquement dans le Labyrinthe, en partie dérobée à sa vue par une palissade, qu'il la retrouve.

L'excitation de la clôture affecte surtout les lieux virtuels de ce texte. La qualité de virtuel, dans le contexte d'une telle fiction narrative, se comprend par opposition aux lieux constitués en points d'ancrage, le hic et nunc des événements narrés. D'autant plus symboliques qu'ils ne sont pas indiciels, ils figurent, comme mis en abyme, les pouvoirs (et les égarements) de l'imagination. La campagne est un de ces creusets des fantasmes. L'érotisation dans le rêve libertin est perçue aux antipodes de la vie mondaine, opposant vrais désirs et contraintes d'une vie de représentation. Une trace discrète apparaît dans notre œuvre lorsque Meilcour regrette amèrement la partie de campagne où il se figure (pour la deuxième fois) Hortense en compagnie de Germeuil :

(22) «Voir Germeuil auprès d'Hortense, et penser que, dans la solitude de la campagne $^{15}$ il trouverait mille moments pour lui dire les choses les plus tendres, était un supplice que je ne pouvais supporter... » (pp. 204-205).

L'éloge des petites maisons par Versac raisonne, du point de vue cynique, le même attrait ${ }^{16}$ :

(23) «N'est-ce pas dans une petite maison qu'on soupe sans scandale tête-à-tête? Et peut-on, sans cette ressource former aujourd'hui un engagement? N'en fait-elle pas même un des premiers articles? Une femme qui se respecte, c'est-à-dire, qui, avec le cœur tendre, ou l'esprit libertin, veut cacher sa faiblesse, ou ses sottises, peut-elle en imposer sans le secours d'une petite maison? Eh! quoi de plus pur, de 
moins interrompu, de plus ignoré, que les plaisirs qu'on y goûte, [...] c'est dans une petite maison qu'on réveille [l'amour], ou qu'on le retrouve... » (pp. 193-194). les ragots et la « lutte des places » qu'il déclenche

(26) « Nous nous mîmes à table; je fis vainement ce que je pus pour être auprès de Mademoiselle de Théville, ou pour éviter du moins Madame de Senanges, rien de tout cela ne me fut possible. Madame de Senanges, dont la résolution était prise, me mit d'autorité entre elle et Versac, qui de son côté ne put parvenir à s'approcher de Mademoiselle de Théville, que sa mère et Madame de Lursay gardaient soigneusement contre lui. » (pp. 153-154).

Aux regards croisés répondent des propos dont l'adresse est biaisée. Ceux qui parlent sont ceux qui n'ont rien à échanger; Philippe Berthier a fait justement observer que ceux que l'on désire (ici Meilcour et Hortense) sont ceux qui se taisent. Les autres, Senanges, Versac, Pranzi ne parlent que pour donner une image d'eux-mêmes, ou pour indirectement détruire celle des autres. Le discours fonctionne sur le mode du trope communicationnel, comme sur une scène de théâtre.

L'espace ainsi habité, occupé par des enjeux relationnels, se présente comme un espace stylisé. C'est bien pour cela qu'il domine la contradiction apparente entre l'effet de réel d'un espace jalonné de lieux connus, familiers au lecteur ${ }^{18}$, et l'extrême abstraction de ce décor, nommé plus que décrit. A côté de la vraisemblance, la dématérialisation des lieux montre combien l'espace est happé par le sens. Là encore comme au théâtre. 
La meilleure illustration de la superposition des genres est une «sortie » de Versac, jouant entre mise en scène narrative et dire effectif théâtralisé, une rude épreuve pour les nerfs de Mme de Lursay, tant les événements narrés collent à la situation présente :

(28) «Par exemple j'étais il n'y a pas longtemps, avec une de ces femmes raisonnables, de ces femmes adroites dont les penchants sont ensevelis sous l'air le plus réservé[...] eh bien ! j'étais avec une prude de cette espèce ; l'amant arriva, l'on le reçut froidement, à peine voulut-on le traiter comme une connaissance; mais pourtant les yeux parlèrent, malgré qu'on en eût; la voix s'adoucit; le petit homme, fort neuf encore, fut embarrassé de la situation; et moi, à qui rien n'échappa, je sortis le plus tôt que je pus, pour l'aller dire à tout le monde » (p. 138).

Sur quoi il se lève en effet! Versac maîtrise "son" monde comme on maîtrise un espace scénique (p. 136) avec faste et calcul; tout à l'opposé Meilcour se perd en chasséscroisés inefficaces, Meilcour qui aux Tuileries est descendu à la mauvaise porte et qui au Pont-Royal n'a pas son équipage pour faire suivre son inconnue, Meilcour qui durant trois jours la cherche partout, sauf là où il l'aurait trouvée, Meilcour enfin complètement dépassé par une situation triangulaire où la joie maligne de mortifier Mme de Lursay en feignant de l'intérêt pour Mme de Senanges, lui fait oublier qu'il parle devant Mlle de Théville!

\section{Conclusion. L'espace et l'initiation.}

58 Je conclurai sur cette théâtralisation car c'est elle qui donne sa forme et son sens à l'acquisition d'une maitrise, qui, en d'autres termes, rend compte de la facture inattendue de ce « roman » d'initiation.

59 J'ai parlé à deux reprises d'interruption de l'histoire, souvent en effet les commentateurs ont souligné son inachèvement. Les parties annoncées dans la Préface, illustrant la dernière étape, celle où le Narrateur, grâce à « une femme estimable » est revenu de sa vie dissolue, ces parties n'existent pas; nous ne saurons jamais avec certitude s'il s'agit d'Hortense. Or en soulignant l'alliance de l'unité de lieu avec un intervalle temporel restreint, j'ai allégué la représentativité de cette tranche de vie, «un prélèvement significatif, comme si la somme de l'initiation figurait déjà dans le fragment mis à plat sous nos yeux.

60 «Mis à plat» précisément. La théâtralisation de la vie mondaine a une visée démonstrative, c'est le dévoilement d'un monde de référence par rapport auquel se marquent des positions : Meilcour y fait ses premiers pas hésitants, Lursay, Senanges, Versac, en sont, avec des statuts nuancés, Hortense, et à certains égards Mme de Théville et Mme de Meilcour, elles, sont au-dessus. La spatialisation fait système, et la stratégie romanesque a joué plus loin même qu'elle ne l'annonçait le jeu de la Comédie, celui du théâtre en général, la transposition de la durée dans l'espace. En faisant monter sur le même plateau les figures de l'initiant, des mondains accomplis qui parviennent à le séduire (mais sans le corrompre tout à fait - Meilcour a des remords) et les figures tutélaires de la vertu, dédaignant les vanités du libertinage ${ }^{19}$, l'auteur syntagmatise la chronologie de l'histoire. Il projette dans l'ordre de la juxtaposition, ce qui était censé appartenir à une succession dans le temps, "les trois âges de la connaissance $»^{20}$. On comprend pourquoi quinze jours ont suffi, et on comprend aussi l'intérêt des coulisses: la Cour que nous ne voyons pas mais que nous pressentons comme la caisse de résonances aux ragots des Versac et Senanges, où Meilcour aura 
aussi ses heures de gloire, la campagne, lieu d'une intimité fantasmée, et la province où Hortense et sa mère ont gardé un ancrage moral.

\section{BIBLIOGRAPHIE}

ADAM Jean-Michel, Le texte narratif, Nathan Université, coll. Fac. Linguistique, Paris 1994.

BERTHIER Philippe, «Le souper impossible », Les Paradoxes du romancier, édités sous la direction de P. Rétat, P.U. de Grenoble 1975, pp. 75-87.

COULET Henri, Le roman jusqu'à la Révolution, A. Colin, Paris, 1967, T. I, pp. 365-373.

FAVRE Robert, «Les mémoires d'un oublieux », Les Paradoxes du romancier, édités sous la direction de P. Rétat, P.U. de Grenoble 1975, pp. 61-74.

GAUBERT Serge, «Synchronie et diachronie ou la naissance du narrateur », op. cit., pp. 43-59.

GAULMYN Pierre de, «Essai sur l'écriture des Égarements », op. cit., pp. 3-16.

GENETTE Gérard, Figures III, Le Seuil 1972.

GÉRAUD Violaine, La Lettre et l'esprit de Crébillon fils, Sedes, « Les livres et les hommes », Paris 1995. HENRIOT Émile, Les Livres du second rayon, Paris, éd. Le Livre, 1926 (rééd. 1948), pp. 177-201.

KIBÉDI-VARGA Aron, « le roman est un anti-roman », Littérature $n^{\circ} 48$, Texte contre-texte, oct. 1982, pp. 3-20.

MICHEL Pierre, «Les Égarements ou le roman impossible », Les Paradoxes du romancier, édités sous la direction de P. Rétat, P.U. de Grenoble 1975, pp. 23-42.

REICHLER Claude, « Le récit d'initiation dans le roman libertin », Littérature $n^{\circ}$ 47, oct. 1982, pp. 100-112.

RÉTAT Pierre, éd. Les Paradoxes du romancier : les « Égarements » de Crébillon, P.U. de Grenoble, 1975. RUSTIN Jacques, « Définition et explication du roman libertin des Lumières », Travaux de linguistique et de littérature de Strasbourg, XVI, 2, 1978, pp. 27-34.

SGARD Jean, « La notion d'égarement chez Crébillon » Dix-Huitième siècle I, 1969, pp. 241-249.

— «L'Incipit des Égarements », Les Paradoxes du romancier, édités sous la direction de P. Rétat, P.U. de Grenoble 1975, pp. 17-21.

TODOROV Tzvetan, Poétique, vol. 2 de « Qu'est-ce que le structuralisme ? », Seuil, « Points », Paris, 1968.

WALD-LASOWSKI Roman, « Crébillon fils et le libertinage galant », Littérature $\mathrm{N}^{\circ}$ 47, oct. 1982, pp. 83-99.

\section{NOTES}

1. Émile Henriot, Les Livres du second rayon, Paris, éd. Le Livre, 1926 (rééd. 1948), pp. 177-201. 
2. La littérature contemporaine s'en est fait l'écho.

3. Je renvoie pour l'explicitation du processus à la synthèse proposée par A. Kibédi-Varga, «le roman est un anti-roman ", Littérature $n^{\circ} 48$, Texte contre-texte, oct. 1982, pp. 3-20.

4. J'adopte, à la fois parce qu'elles sont connues et largement pertinentes, les catégories de G. Genette (Figures III, Le Seuil 1972), où histoire désigne le signifié (le contenu narré), récit le signifiant (le support, le texte narratif), et narration l'acte narratif, l'acte producteur.

5. J. Sgard, «L'Incipit des Égarements », Les Paradoxes du romancier, édités sous la direction de P. Rétat, P.U. de Grenoble 1975, pp. 17-21.

6. L'édition de référence est ici celle de Jean Dagen, GF-Flammarion, Paris, 1985.

7. Dans ses Lettres au Marquis de Sévigné, ces mêmes lettres furent parfois attribuées à Crébillon, Mémoires sur la vie de Mademoiselle de Lenclos, par Mr B***, Amsterdam, 1763, cité par Cl. Reichler, $1982: 103$.

8. Au sens strict du terme, ce qui advient et offre matière à récit: épurée, "réduite à une esquisse ", comme l'observe avec justesse Violaine Géraud (1995, p. 206), l'action dans ce roman a pour temps fort le coup de foudre à l'Opéra. C'est la séquence où trouve place la canonique " perturbation " (Todorov 1968, Adam, 1994) justifiant intermittences et " égarements » à suivre. 9. Ce discours d'initiation est souvent appelé dans les études crébilloniennes «le discours de l'Étoile».

10. La rencontre de Marianne et de Valville, par la grâce d'une entorse !

11. Celui du roman libertin mondain par opposition au roman libertin pornographique (cf. $\mathrm{H}$. Coulet, Le roman jusqu'à la Révolution, A. Colin, Paris, 1967, T. I, pp. 365-373). Évidemment, les rapports entre choix des lieux et choix d'un langage mériteraient eux-mêmes une analyse approfondie.

12. La leçon de l'Étoile significativement est reçue juste avant la résolution de l'aventure-Lursay.

13. Mme de Meilcour est obligée de recevoir Versac, qu'elle n'aime pas, Mme de Lursay, mortifiée, ne peut faire autrement que d'accueillir à sa table Pranzi, amené par le même Versac.

14. Embrayeur spatial, il renvoie à l'instance énonciative, naturellement dominante dans ce roman... tout en discours.

15. Pour une analyse de la fonction érotisante de la campagne, du "loin du fracas des villes », voir le commentaire de Roman Wald-Lasowski, sur le Sylphe de «Crébillon dans Crébillon fils et le libertinage galant ", Littérature $\mathrm{N}^{\circ} 47$, oct. 1982, pp. 83-99.

16. L'intérêt porté aux "petites maisons » dans l'imaginaire libertin, leur fonction séductrice, ont été mis en lumière par la conférence de M. Delon, La visite de la maison: Bastide (1758), Mario Praz (1979),Séville, 7 mars 1997.

17. Cf. Philippe Berthier, « Le souper impossible », op. cit., pp. 75-87.

18. Alors que Crébillon peut sacrifier aussi à la fantaisie orientale, à la féerie.

19. Hortense s'est montrée de marbre face au charme « irrésistible » de Versac.

20. Cf. Serge Gaubert, "Synchronie et diachronie ou la naissance du narrateur », op. cit., pp. 43-59.

\section{AUTEUR}

ANNA JAUBERT

Université de Nice-Sophia Antipolis 